\title{
Autonomous Hovering of a Noncyclic Tiltrotor UAV: Modeling, Control and Implementation
}

\author{
A. Sanchez, J. Escareño, O. Garcia and R. Lozano \\ Heudiasyc-UTC UMR 6599 Centre de Recherches de Royallieu B.P. \\ 2052960205 Compiegne France Tel.: + $33(0) 344234423$; fax: +33 \\ (0)3442344 77 (e-mail: \{asanchez, jescaren, ogarcias, \\ rlozano\}@hds.utc.fr)
}

\begin{abstract}
The aim of this paper is to present a mini tilt-rotor unmanned aerial vehicle which is capable to perform hover flight. Unlike conventional full-scale tiltrotors, in our design we avoid the use of swashplate and we propose a simpler mechanical design which use only the tilting rotors to stabilize the vehicle dynamics. A detailed mathematical model is derived via the Newton-Euler formalism. A nonlinear control scheme, incorporating bounded smooth function, is obtained from the decoupled dynamics and applied to real prototype for controlling hover flight.
\end{abstract}

\section{INTRODUCTION}

Nowadays, the Unmanned Aerial Vehicle (UAV) developments represent an appealing research area due to its widerange application field. In terms of military application it might be mentioned: hostile zone reconnaissance, hazardous biological or chemical agent detection, etc. Within civilian field, we can cite the natural disaster support, assistance for earth science research, agricultural support, etc.

Conventional tiltrotor vehicles, such as the Bell Boeing V22 Osprey, are mechanically complex systems since it employs a swashplate and differential rotor tilting to control pitch and yaw, respectively. This represents a drawback in terms of maintenance and replacement cost. Moreover, these vehicles may not be a handy tool for repetitive test flights due to its crash vulnerability. For these reasons we propose a configuration, called T-Phoenix (see Fig. 1), which copes with the pitch-yaw motion using a simpler mechanical system based on rotors (noncyclic propellers) tilting. This paper describes the first stage of an undergoing project to develop an aircraft that blends the vertical lift capability of a helicopter with the horizontal flight performance of an airplane. This kind of vehicles does not requires a run-way or an auxiliary launch/recovering device, such as catapultes or parachutes, because of its vertical take-off/landing capabilities.

In Gress [2002] and Gress [2003] the author proposes and implements a relative complex configuration to carry out hover flight taking advantage of the gyroscopic effect provided by the tilting rotors. It is worth pointing up that driving the pitch angle, represents a challenge because the contribution of gyroscopic-based on torque is not strong enough to cope with the pitch dynamics. In Kendoul et al. [2002] the authors present the mathematical model and simulation results of a backstepping algorithm in order to stabilize a tilt-rotor vehicle inspired by Gress's mechanism.
The main contribution of this paper is the design and implementation of a simple tilting mechanism to perform hover flight. In addition, we present a detailed mathematical model based on the Newton-Euler formulation and we propose a control strategy that consists in decoupling the 6-DOF dynamics in three independent subsystems, simplifying the control task. It is also proposed a nonlinear control algorithm to deal with a chain of integrators using bounded smooth functions.

The paper outline is: Section 2 provides the description of the T-Phoenix and also presents the equations that models the translational and rotational motion. The controller design which stabilizes the dynamics of the UAV is presented in section 3. Section 4 reports the experimental setup and depicts the experimental results. Finally some concluding remarks and perspectives are given in section 5 .

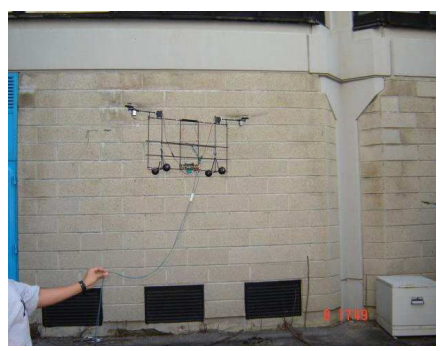

Fig. 1. T-Phoenix autonomous hovering

\section{T-PHOENIX UAV}

Before providing the dynamical equations of the TPhoenix UAV, it results interesting to illustrate the way as the UAV drives the attitude based on rotors tilting.

Roll motion and Altitude: The roll motion of the vehicle is regulated by the difference in the angular velocity of rotors (see Fig. 2). The altitude is controlled by increasing or decreasing the thrust of the rotors. 


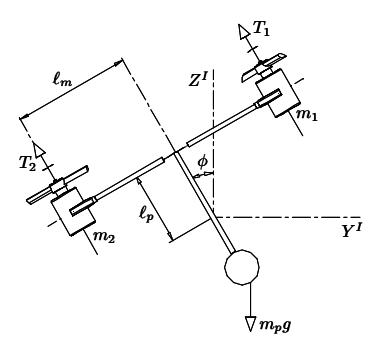

Fig. 2. Roll motion and altitude.

Pitch motion: The advantage of this configuration is the addition of an extra mass, besides the ones of the rotors, which is placed down enough to provide a weightbased torque, obtaining then, a pendular damped effect (naturally stable), see Fig. 3. To counteract this pendular motion, the rotors tilt parallel (at the same time) in opposite sense of the pitch motion maintaining the upwards position, emulating a mobile pivot, and moreover behaving as damping factor.

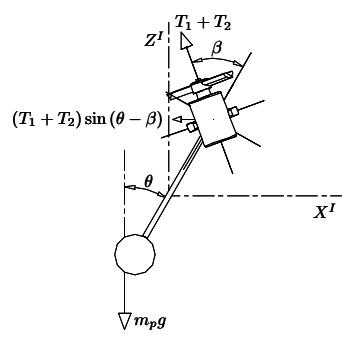

Fig. 3. Pitch motion.

Yaw motion: The yaw motion is driven via the rotors' differential tilting generating the required torque to provoke a rotation (see Fig. 4).

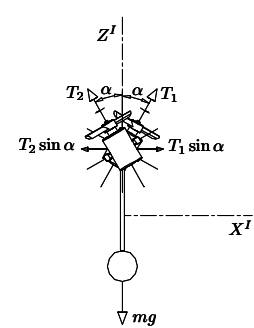

(a)

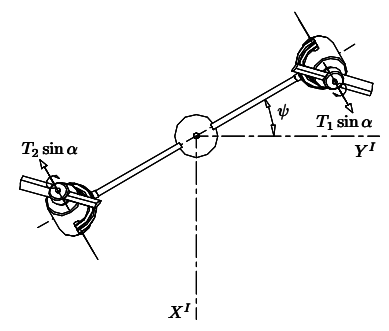

(b)
Fig. 4. Yaw motion.

\subsection{Dynamical model}

Let $\mathcal{I}=\left\{i_{x}^{\mathcal{I}}, j_{y}^{\mathcal{I}}, k_{z}^{\mathcal{I}}\right\}$ denote the right handed inertial frame, $\mathcal{B}=\left\{i_{x}^{\mathcal{B}}, j_{y}^{\mathcal{B}}, k_{z}^{\mathcal{B}}\right\}$ denotes frame attached to the body's aircraft whose origin is located at its center of gravity (see Fig. 5). Two auxiliary frames are obtained from the tilting motion. The first tilting, to drive the yaw motion, produces the frames $\mathcal{Y}_{1}=\left\{i_{x}^{\mathcal{Y}_{1}}, j_{y}^{\mathcal{Y}_{1}}, k_{z}^{\mathcal{Y}_{1}}\right\}$ and $\mathcal{Y}_{2}=\left\{i_{x}^{\mathcal{Y}_{2}}, j_{y}^{\mathcal{Y}_{2}}, k_{z}^{\mathcal{Y}_{2}}\right\}$. Afterwards, the frame $\mathcal{P}=\left\{i_{x}^{\mathcal{P}}, j_{y}^{\mathcal{P}}, k_{z}^{\mathcal{P}}\right\}$ appears at regulating the pitch motion.

Let the vector $\mathbf{q}=(\xi, \eta)^{T}$ denotes the generalized coordinates where $\xi=(x, y, z)^{T} \in \Re^{3}$ denotes the translation coordinates relative to the inertial frame, and $\eta=$ $(\psi, \theta, \phi)^{T} \in \Re^{3}$ describes the vehicle orientation expressed

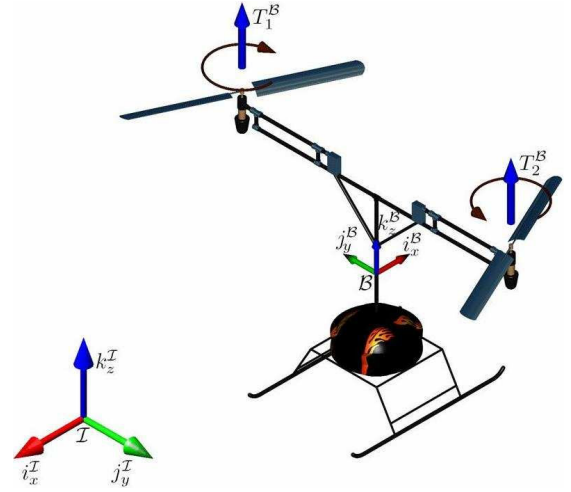

Fig. 5. T-Phoenix model.

in the classical yaw, pitch and roll angles (Euler angles). The orientation of the convertible UAV is given by the orthonormal rotation matrix $R^{\mathcal{B} \rightarrow \mathcal{I}}$.

$$
R^{\mathcal{B} \rightarrow \mathcal{I}}=\left(\begin{array}{ccc}
c_{\theta} c_{\psi} & s_{\phi} s_{\theta} c_{\psi}-c_{\phi} s_{\psi} & c_{\phi} s_{\theta} c_{\psi}+s_{\phi} s_{\psi} \\
c_{\theta} s_{\psi} & s_{\phi} s_{\theta} s_{\psi}+c_{\phi} c_{\psi} & c_{\phi} s_{\theta} s_{\psi}-s_{\phi} c_{\psi} \\
-s_{\theta} & s_{\phi} c_{\theta} & c_{\phi} c_{\theta}
\end{array}\right)
$$

where $s_{a}=\sin (a)$ and $c_{a}=\cos (a)$.

Regulating yaw, implies the knowledge of two matrices, since the vehicle tilts differentially, these matrices are given by

$$
R^{\mathcal{Y}_{1} \rightarrow \mathcal{B}}=\left(\begin{array}{ccc}
c_{\alpha} & 0 & s_{\alpha} \\
0 & 1 & 0 \\
-s_{\alpha} & 0 & c_{\alpha}
\end{array}\right), \quad R^{\mathcal{Y}_{2} \rightarrow \mathcal{B}}=\left(\begin{array}{ccc}
c_{\alpha} & 0 & -s_{\alpha} \\
0 & 1 & 0 \\
s_{\alpha} & 0 & c_{\alpha}
\end{array}\right)
$$

Notice that both matrices tilt about $j_{y}^{\mathcal{B}}$ axis.

The associated matrix to the pitch control is written as

$$
R^{\mathcal{P} \rightarrow \mathcal{B}}=\left(\begin{array}{ccc}
c_{\beta} & 0 & -s_{\beta} \\
0 & 1 & 0 \\
s_{\beta} & 0 & c_{\beta}
\end{array}\right)
$$

The Newton-Euler formulation provides the overall motion equations of a rigid body, which are given by the following expressions

$$
\begin{aligned}
\bar{m} \dot{V}^{\mathcal{B}}+\Omega \times \bar{m} V^{\mathcal{B}} & =F^{\mathcal{B}} \\
\mathbf{I} \dot{\Omega}+\Omega \times \mathbf{I} \Omega & =\Gamma^{\mathcal{B}}
\end{aligned}
$$

where $F^{\mathcal{B}} \in \Re^{3}$ and $\Gamma^{\mathcal{B}} \in \Re^{3}$ are, respectively, the total force and torque applied to the aircraft's center of gravity $(C G), \bar{m}=\operatorname{diag}(m) \in \Re^{3 \times 3}, m \in \Re$ denotes the vehicle's mass, $\Omega=(p, q, r)^{T}$ is the body frame angular velocity, $V^{\mathcal{B}}=(u, v, w)^{T}$ is the translational velocity of the aircraft's center of mass, $\mathbf{I} \in \Re^{3 \times 3}$ contains the moments of inertia about $\mathcal{B}$.

Translational motion In this subsection, we derive the translational and rotational dynamics, via the NewtonEuler formalism.

Body frame: The tilting motion splits the thrust up in several components, affecting the translational and rotational motion. The following transformation provides the total rotors' thrust in terms of the body frame

$$
\begin{aligned}
& T_{1}^{\mathcal{B}}=R^{\mathcal{Y}_{1} \rightarrow \mathcal{B}} R^{\mathcal{P} \rightarrow \mathcal{Y}_{1}} T_{1}^{\mathcal{P}} \\
& T_{2}^{\mathcal{B}}=R^{\mathcal{Y}_{2} \rightarrow \mathcal{B}} R^{\mathcal{P} \rightarrow \mathcal{Y}_{2}} T_{2}^{\mathcal{P}}
\end{aligned}
$$


the translation motion of the UAV is described by the following vectorial equation

$$
\bar{m} \dot{V}^{\mathcal{B}}+\Omega \times \bar{m} V^{\mathcal{B}}=R^{\mathcal{I} \rightarrow \mathcal{B}} m G^{\mathcal{I}}+T_{1}^{\mathcal{B}}+T_{2}^{\mathcal{B}}
$$

where $G^{\mathcal{I}} \in \Re^{3}, G^{\mathcal{I}}=(0,0,-g)$ is the gravity vector.

Inertial frame: The translation dynamics relative to the inertial frame is provided by the following expression

$$
\left\{\begin{array}{l}
V^{\mathcal{I}}=\dot{\xi} \\
m \dot{V}^{\mathcal{I}}=m G^{\mathcal{I}}+R^{\mathcal{B} \rightarrow \mathcal{I}}\left(T_{1}^{\mathcal{B}}+T_{2}^{\mathcal{B}}\right)
\end{array}\right.
$$

Rotational motion The RHS terms of (2) correspond to the applied torques on the rigid-body, involving the ones provided by the propeller, these are described next:

Actuators torque: The actuators torque is given by

$$
\Gamma_{c}=\ell_{m 1}^{\mathcal{B}} \times T_{1}^{\mathcal{B}}+\boldsymbol{\ell}_{m 2}^{\mathcal{B}} \times T_{2}^{\mathcal{B}}
$$

where $\ell_{m 1}^{\mathcal{B}}=\left(0, \ell_{m}, \ell_{p}\right), \ell_{m 2}^{\mathcal{B}}=\left(0,-\ell_{m}, \ell_{p}\right)$ are the distances from the $C G$ to the rotors.

\section{Weight torque:}

The torque provided by the pendular mass

$$
\Gamma_{p}=\ell_{p}^{\mathcal{B}} \times R^{I \rightarrow \mathcal{B}} m G^{\mathcal{I}}
$$

where $\boldsymbol{\ell}_{p}^{\mathcal{B}}=\left(0,0,-\ell_{p}\right)$

Gyroscopic torque: In the yaw control, the gyroscopic terms caused by tilting rotors may be modeled as

$$
\left\{\begin{array}{l}
\Gamma_{G_{\alpha 1}}^{\mathcal{Y}_{1}}=I_{p}\left(V_{\alpha 1}^{\mathcal{Y}_{1}} \times \omega_{p 1}^{\mathcal{Y}_{1}}\right) \\
\Gamma_{G_{\alpha 2}}^{\mathcal{Y}_{2}}=I_{p}\left(V_{\alpha 2}^{\mathcal{Y}_{2}} \times \omega_{p 2}^{\mathcal{Y}_{2}}\right)
\end{array}\right.
$$

and the one provided by the pitch regulation is

$$
\Gamma_{G_{\beta}}^{\mathcal{P}}=\sum_{i=1}^{2} I_{p}\left(V_{\beta}^{\mathcal{P}} \times \omega_{p i}^{\mathcal{P}}\right)
$$

where $V_{\alpha 1}^{\mathcal{Y}_{1}}=(0, \dot{\alpha}, 0)^{T}, V_{\alpha 2}^{\mathcal{Y}_{2}}=(0,-\dot{\alpha}, 0)^{T}, \omega_{p 1}^{\mathcal{Y}_{1}}=$ $\left(0,0, \omega_{p 1}\right)$ and $\omega_{p 2}^{\mathcal{Y}_{2}}=\left(0,0, \omega_{p 2}\right)$. The final expression that models the total gyroscopic torque relative to the body frame can be written as

$$
\Gamma_{G}^{\mathcal{B}}=R^{\mathcal{P} \rightarrow \mathcal{B}} \Gamma_{G_{\beta}}^{\mathcal{P}}+R^{\mathcal{Y}_{1} \rightarrow \mathcal{B}} \Gamma_{G_{\alpha 1}}^{\mathcal{Y}_{1}}+R^{\mathcal{Y}_{2} \rightarrow \mathcal{B}} \Gamma_{G_{\alpha 2}}^{\mathcal{Y}_{2}}
$$

where $I_{p}$ is the inertia moment of the propeller.

Drag torque: Finally, considering the same blade's geometry, the drag torque may be modeled as

$\Gamma_{D}^{\mathcal{B}}=\ell_{D}^{\mathcal{B}} \times\left(R^{\mathcal{Y}_{1} \rightarrow \mathcal{B}} R^{\mathcal{P} \rightarrow \mathcal{B}} D_{1}^{\mathcal{P}}\right)+\ell_{D}^{\mathcal{B}} \times\left(R^{\mathcal{Y}_{2} \rightarrow \mathcal{B}} R^{\mathcal{P} \rightarrow \mathcal{B}} D_{2}^{\mathcal{P}}\right)$

where $D_{1}^{\mathcal{P}}=k_{D} \omega_{p 1}^{2}$ and $D_{2}^{\mathcal{P}}=k_{D} \omega_{p 2}^{2}$ denote the drag force of each propeller. $k_{D}$ represents the blade's aerodynamic parameters, involving the blade profile, surface and pitch and $\ell_{D}^{\mathcal{B}}$ denotes the distance from the vehicle's gravity center to the point where the drag is exerted.

Reduced model For further control analysis, let us shape a reduced model by introducing the following assumptions and facts:

A1. The gyroscope effect $\left(\Gamma_{G_{\alpha 1}}^{\mathcal{Y}_{1}}\right.$ and $\left.\Gamma_{G_{\alpha 2}}^{\mathcal{Y}_{2}}\right)$ generated during the counter-rotating propellers combined with differential tilt of the rotors is self-compensated.

A2. During the pitch control, the inherent gyroscopic caused by the slight tilting rotors is disregard.
A3. We have normalized the inertial tensor matrix $\mathbf{I}$ and the vehicle mass $m$.

Based on the previous considerations, we obtain

$$
\begin{aligned}
\ddot{x}= & T c_{\alpha} c_{\beta} s_{\psi} s_{\phi}+T c_{\alpha} c_{\beta} c_{\psi} s_{\theta} c_{\phi} \\
& +\frac{u_{\phi}}{\ell_{m}} s_{\alpha} s_{\beta} s_{\psi} s_{\phi}+\frac{u_{\phi}}{\ell_{m}} s_{\alpha} s_{\beta} c_{\psi} s_{\theta} c_{\phi} \\
& -T c_{\alpha} s_{\beta} c_{\psi} c_{\theta}+T s_{\alpha} c_{\beta} c_{\psi} c_{\theta} \\
\ddot{y}= & -T c_{\alpha} c_{\beta} c_{\psi} s_{\phi}+T c_{\alpha} c_{\beta} s_{\psi} s_{\theta} c_{\phi} \\
& -\frac{u_{\phi}}{\ell_{m}} s_{\alpha} s_{\beta} c_{\psi} s_{\phi}+\frac{u_{\phi}}{\ell_{m}} s_{\alpha} s_{\beta} s_{\psi} s_{\theta} c_{\phi} \\
& -T c_{\alpha} s_{\beta} s_{\psi} c_{\theta}-\frac{u_{\phi}}{\ell_{m}} s_{\alpha} c_{\beta} s_{\psi} c_{\theta} \\
\ddot{z}= & T c_{\alpha} s_{\beta} s_{\theta}+T c_{\alpha} c_{\beta} c_{\theta} c_{\phi} \\
& -\frac{u_{\phi}}{\ell_{m}} s_{\alpha} c_{\beta} s_{\theta}+\frac{u_{\phi}}{\ell_{m}} s_{\alpha} s_{\beta} c_{\theta} c_{\phi}-g \\
\ddot{\psi}= & u_{\phi} c_{\alpha} s_{\beta}-T \ell_{m} c_{\beta} s_{\alpha} \\
\ddot{\theta}= & \frac{u_{\phi}}{\ell_{m}} \ell_{p} c_{\beta} s_{\alpha}-T \ell_{p} c_{\alpha} s_{\beta}-g \ell_{p} s_{\theta} \\
\ddot{\phi}= & u_{\phi} c_{\alpha} c_{\beta}+T \ell_{m} s_{\alpha} s_{\beta}-g \ell_{p} c_{\theta} s_{\phi}
\end{aligned}
$$

where $u_{\phi}=\ell_{m}\left(T_{1}-T_{2}\right)$.

In order to simplify the control task, we have split the 6-DOF dynamics up in three subdynamics, which are:

\section{Lateral dynamics:}

This dynamics $(\theta=0, \psi=0, \alpha=0, \beta=0)$ arises from regulating the roll angle $\phi$, this leads us to the following scalar expressions:

$$
\left\{\begin{array}{l}
\ddot{y}=-T \sin \phi \\
\ddot{z}=T \cos \phi-g \\
\ddot{\phi}=u_{\phi}-g \ell_{p} \sin \phi
\end{array}\right.
$$

\section{Longitudinal dynamics:}

The result of controlling the pitch angle $\theta$ is the longitudinal dynamics $(\phi=0, \psi=0, \alpha=0)$, which is described by

$$
\left\{\begin{array}{l}
\ddot{x}=T \sin (\theta-\beta) \\
\ddot{\theta}=-g \ell_{p} \sin \theta-T \ell_{p} \sin \beta
\end{array}\right.
$$

where $\beta$ obeys to the pitch control input $u_{\theta}$ behavior.

\section{Axial dynamics:}

The remaining dynamics $(\theta=0, \phi=0, \beta=0)$ is driven by the differential tilt of the rotors at it may written as

$$
\left\{\ddot{\psi}=-T \ell_{m} \sin \alpha\right.
$$

\section{CONTROL ALGORITHM DESIGN}

In this section we present the control strategy to drive the UAV in hover flight. To simplify we deal separately the lateral, longitudinal and axial dynamics.

To handle the system dynamics we get the corresponding linear approximation of (15), therefore we obtain 


$$
\left\{\begin{array}{l}
\ddot{x}=T \theta-T \beta \\
\ddot{y}=-\phi T \\
\ddot{z}=T-g \\
\ddot{\psi}=-T \ell_{m} \alpha \\
\ddot{\theta}=-g \ell_{p} \theta-T \ell_{p} \beta \\
\ddot{\phi}=-g \ell_{p} \phi+u_{\phi}
\end{array}\right.
$$

First, we tackle the altitude dynamics, proposing the following control input

$$
T=-k_{1} \dot{z}-k_{2} z+g
$$

where $k_{1}$ and $k_{2}$ are positive constants. Then, altitude dynamics is given by

$$
\ddot{z}=-k_{1} \dot{z}-k_{2} z
$$

Choosing $k_{1}$ and $k_{2}$ such that $s^{2}+k_{1} s+k_{2}$ is a Hurwitz polynomial, therefore $\dot{z} \rightarrow 0$ and $z \rightarrow 0$ as $t \rightarrow \infty$. This implies that there exists a time large enough $T_{1}$ such that

$$
T=g
$$

for all $t>T_{1}$. From the latter, we get

$$
\begin{aligned}
& \ddot{x}=g \theta-g \beta \\
& \ddot{y}=-\phi g \\
& \ddot{\psi}=-g \ell_{m} \alpha \\
& \ddot{\theta}=-g \ell_{p} \theta-g \ell_{p} \beta \\
& \ddot{\phi}=-g \ell_{p} \phi+u_{\phi}
\end{aligned}
$$

Similarly as the altitude dynamics control, the axial dynamics is stabilized with the following controller

$$
\alpha=k_{3} \dot{\psi}+k_{4} \psi
$$

where $k_{1}$ and $k_{2}$ are positive constants. Then, equation (21) becomes

$$
\ddot{\psi}=-g \ell_{m} k_{3} \dot{\psi}-g \ell_{m} k_{4} \psi
$$

Now, defining $k_{3}$ and $k_{4}$ such that $s^{2}+g \ell_{m} k_{3} s+g \ell_{m} k_{4}$ is a Hurwitz polynomial, hence $\dot{\psi} \rightarrow 0$ and $\psi \rightarrow 0$ as $t \rightarrow \infty$. Theorem 3.1. The origin of the closed-loop system composed by $(20)$, (23) with the bounded smooth functionbased control input

$u_{\phi}=g \ell_{p} \phi-\tanh (\dot{\phi})-a \tanh \left(z_{1}\right)+b \tanh \left(z_{2}\right)+c \tanh \left(z_{3}\right)$

where

$$
\begin{aligned}
z_{1} & =\phi+\dot{\phi} \\
z_{2} & =a \dot{y}-g z_{1}-a g \phi \\
z_{3} & =a b g y+z_{2}+b g \dot{y}+a b g \dot{y}-b g^{2} \phi \\
a+b+c & <1 . \\
b+c & <a \\
0 & <c<b
\end{aligned}
$$

is asymptotically stable.

Proof 3.2. We propose $u_{\phi}$ as

$$
u_{\phi}=g \ell_{p} \phi-\tanh (\dot{\phi})-\eta_{1}
$$

where $\eta_{1}$ will be deduce later satisfying $\left|\eta_{1}\right| \leq l_{\eta_{1}}<1$ with $l_{\eta_{1}}$ is a positive constant. From now on, functions $\eta_{i}$ and constants $l_{\eta_{i}}>0$ for $i=2,3$ will be obtained throughout the control synthesis.

Introducing (26) into (23) we obtain

$$
\ddot{\phi}=-\tanh (\dot{\phi})-\eta_{1}
$$

Let

$$
V_{1}=\ln (\cosh (\dot{\phi}))
$$

then

$$
\dot{V}_{1}=-\tanh (\dot{\phi})\left(\tanh (\dot{\phi})+\eta_{1}\right)
$$

Note that if $|\dot{\phi}|>l_{\eta_{1}}$ implies $\dot{V}_{1}<0$. Thus, there exists a time $T_{2}>0$ such that

$$
|\dot{\phi}| \leq l_{\eta_{1}} \forall t>T_{2}
$$

Therefore equation $(23)$ reduces to

$$
\ddot{\phi}=-\dot{\phi}-\eta_{1}
$$

Let us define

$$
z_{1}=\phi+\dot{\phi}
$$

From (29) and (30) we get

$$
\dot{z}_{1}=-\eta_{1}
$$

Let us define $\eta_{1}$ as

$$
\eta_{1}=a \tanh \left(z_{1}\right)+\eta_{2}
$$

where $a$ is a positive constant, $\left|\eta_{2}\right| \leq l_{\eta_{2}}<a$ and $a+$ $l_{\eta_{2}}<1$.

Let us propose

$$
V_{2}=\ln \left(\cosh \left(z_{1}\right)\right)
$$

then

$$
\dot{V}_{2}=-\tanh \left(z_{1}\right)\left(a \tanh \left(z_{1}\right)+\eta_{2}\right)
$$

If $\left|z_{1}\right|>l_{\eta_{2}}$ implies $\dot{V}_{2}<0$. Thus, there exists a time $T_{3}>T_{2}$ such that

$$
\left|z_{1}\right| \leq l_{\eta_{2}} \forall t>T_{3}
$$

and therefore equation $(31)$ reduces to

$$
\dot{z}_{1}=-a z_{1}-\eta_{2} \forall t>T_{3}
$$

From (30) we obtain for $t>T_{3}$

$$
\phi(t)=e^{-\left(t-T_{2}\right)} \phi\left(T_{2}\right)+\int_{T_{2}}^{t} e^{-(t-\tau)} z_{1}(\tau) d \tau
$$

Thus it follows that for a time $T_{4}$ large enough we obtain

$$
|\phi(t)| \leq l_{\eta_{2}}+\delta
$$

for some $\delta$ arbitrarily small.

To establish a bound of $\dot{y}$ let us define

$$
z_{2}=a \dot{y}-g z_{1}-a g \phi
$$

then from (29), (20) and (35) we obtain

$$
\dot{z}_{2}=g \eta_{2}
$$

Let us define $\eta_{2}$ as

$$
\eta_{2}=-b \tanh \left(z_{2}\right)-\eta_{3}
$$

where $b$ is a positive constant, $\left|\eta_{3}\right| \leq l_{\eta_{3}}<b$ and $b+l_{\eta 3}<a$. Let

$$
V_{3}=\ln \left(\cosh \left(z_{2}\right)\right)
$$


then

$$
\dot{V}_{3}=-g \tanh \left(z_{2}\right)\left(b \tanh \left(z_{2}\right)+\eta_{3}\right)
$$

Note that if $\left|z_{2}\right|>l_{\eta_{3}}$ implies $\dot{V}_{3}<0$. Hence, there exists a time $T_{4}>T_{3}$ such that

$$
\left|z_{2}\right| \leq l_{\eta_{3}} \forall t>T_{4}
$$

therefore the equation (37) reduces

$$
\dot{z}_{2}=-b g z_{2}-g \eta_{3}
$$

To deduce a bound of $y$ consider

$$
z_{3}=a b g y+z_{2}+b g \dot{y}+a b g \dot{y}-b g^{2} \phi
$$

From $(29),(41),(35)$ and (20) we obtain

$$
\dot{z}_{3}=-g \eta_{3}
$$

Proposing $\eta_{3}$ as

$$
\eta_{3}=c \tanh \left(z_{3}\right)
$$

where $c<b$ is a positive constant.

Let

$$
V_{4}=\ln \left(\cosh \left(z_{3}\right)\right)
$$

then

$$
\dot{V}_{4}=-g c \tanh ^{2}\left(z_{3}\right)
$$

which is negative definite for all $z_{3}$. This implies that $z_{3} \rightarrow 0$ as $t \rightarrow \infty$.

From (30) - (44) we deduce that $z_{i} \rightarrow 0$ for $i=1,2$. From $(27),(28),(30),(32)(38)$ and (44) we get that $\dot{\phi} \rightarrow 0$ and $\phi \rightarrow 0$. From (36) and (42) it is follows that $\dot{y} \rightarrow 0$ and $y \rightarrow 0$. This concludes the proof.

In order to stabilize the remaining dynamics (19) and (22) we propose the following state equations

$$
\left(\begin{array}{l}
\xi_{1} \\
\xi_{2} \\
\xi_{3} \\
\xi_{4}
\end{array}\right)=\left(\begin{array}{c}
x \\
\dot{x} \\
\theta \\
\dot{\theta}
\end{array}\right)
$$

The dynamic equations then are given by

$$
\dot{\xi}=A \xi+B \beta
$$

where

$$
\begin{gathered}
A=\left(\begin{array}{cccc}
0 & 1 & 0 & 0 \\
0 & 0 & g & 0 \\
0 & 0 & 0 & 1 \\
0 & 0 & -g \ell_{p} & 0
\end{array}\right) \\
B=\left(\begin{array}{c}
0 \\
-g \\
0 \\
-g \ell_{p}
\end{array}\right)
\end{gathered}
$$

Proposing

$$
\beta=-\bar{k} \xi
$$

where

Thus

$$
\bar{k}=\left(\begin{array}{llll}
\bar{k}_{1} & \bar{k}_{2} & \bar{k}_{3} & \bar{k}_{4}
\end{array}\right)
$$

$$
\dot{\xi}=(A-B \bar{k}) \xi
$$

Choosing $\bar{k}$ such that $(A-B \bar{k})$ is Hurwitz implies that $\xi$ $\rightarrow 0$ as $t \rightarrow \infty$.

\section{EXPERIMENTAL PLATFORM}

In this section, we present the real-time experimental results obtained when applying the proposed controllers, (24), (18), (25) and (47) to the T-Phoenix UAV (see Figures 6).

\subsection{Real-time PC-Control System (PCCS)}

The control is performed trough a real-time simulink XPC target toolbox. The radio and the target $\mathrm{PC}$ are connected using data acquisition cards. The connection in the radio is directly made to the joystick potentiometers for the collective, yaw, pitch, and roll controls.

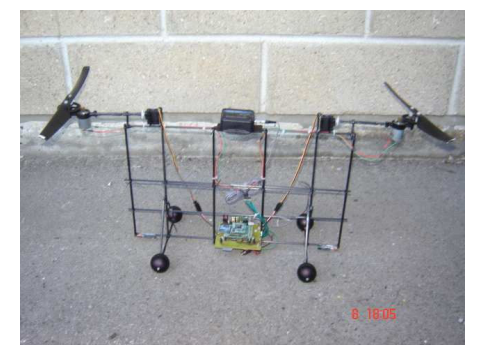

Fig. 6. The T-Phoenix UAV.

Sensors and communication hardware In order to stabilize the helicopter and acquire the system data, we have implemented two different platforms. The first one is devoted to compute the onboard control algorithm for the autonomous attitude-stabilized flight, and the second one to provide the $3 \mathrm{D}$ position feedback for the regulation of the helicopter.

Sensors

- Inertial Measurement Unit (IMU): We have used a 3DM-X1 microstrain IMU which provides the angular rate $(\dot{\psi}, \dot{\phi}, \dot{\theta})$ and the angular position $(\psi, \phi, \theta)$. This IMU combines three angular rate gyros with three orthogonal accelerometers, three orthogonal magnetometers, multiplexer and 16 bit A/D converter.

- Position Measurement Unit (PMU): The vehicle's position $(x, y, z)$ is obtained through a magnetic threedimensional tracker Polhemus. This sensor is very sensitive to electromagnetic noise and has a range of $1.52 \mathrm{~m}$ approximately. For this reason we placed it quite away from the electric motors.

\section{Communication hardware}

The PCCS requests (polling mode) the sensors information via the RS-232 serial communication. The best sensor performance, in terms of transmission data, was obtained at a sampling frequency of $50 \mathrm{~Hz}$. The inertial data is transmitted via a wireless X-Bee modem whereas the Polhemus is directly plugged to the PCCS.

\subsection{Experimental results}

The gain values used for the control law are shown in Table 1. The control goal is to reach the zero attitude $(\psi, \theta, \phi)=$ $(0,0,0)$ and a $3 \mathrm{D}$ desired position $(x, y, z)=(0,0,15 \mathrm{~cm})$, resulting in a autonomous stabilized-hover flight. 

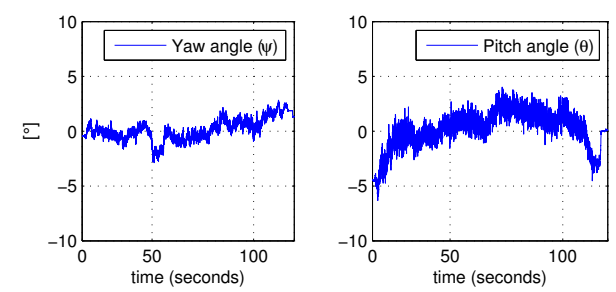

Fig. 7. Yaw and pitch angles.
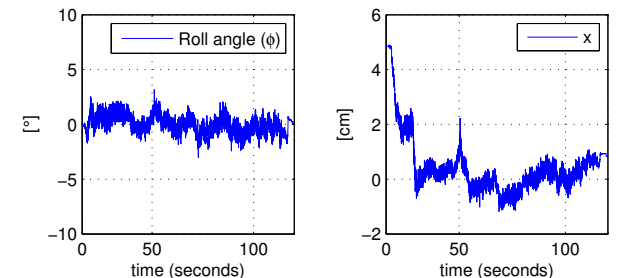

Fig. 8. Roll angle and $x$ position.
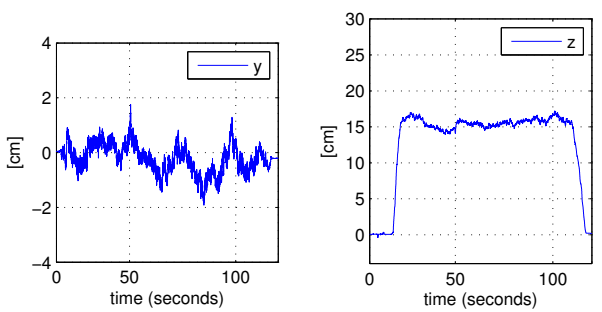

Fig. 9. $x$ and $y$ positions.
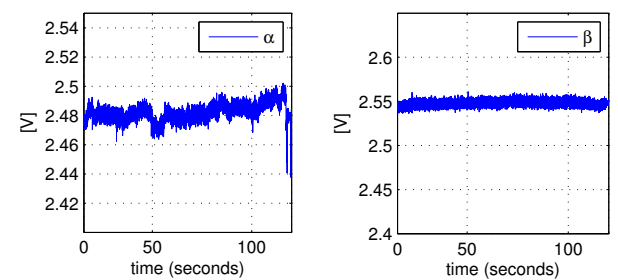

Fig. 10. $\alpha$ and $\beta$ controllers

Figures 7-11 show the performance of the controller during the T-Phoenix's hover flight. As can be seen from Figures 7-11 the proposed control performs well in practice even in presence of significant disturbances.

\begin{tabular}{|c|c|}
\hline \hline Control parameter & Value \\
\hline \hline$k_{1}$ & 1.975 \\
$k_{2}$ & 0.07 \\
$k_{3}$ & 0.001 \\
$k_{4}$ & 0.002 \\
$a$ & 0.4 \\
$b$ & 0.2 \\
$c$ & 0.1 \\
$\bar{k}_{1}$ & 0.002 \\
$\bar{k}_{2}$ & 0.00001 \\
$\bar{k}_{3}$ & 0.04 \\
$\bar{k}_{4}$ & 0.00003 \\
\hline \hline
\end{tabular}

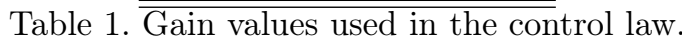
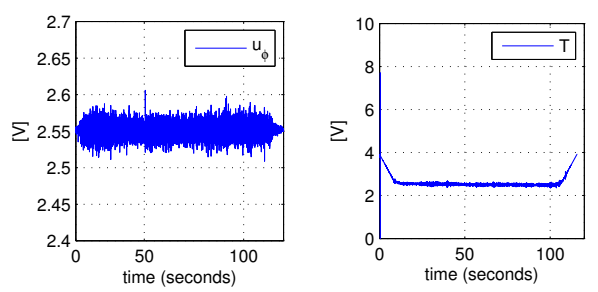

Fig. 11. $u_{\phi}$ control and throttle input.

\section{CONCLUSION}

A noteworthy accomplishment is the autonomous hover flight of the mini-tiltrotor UAV (T-Phoenix) employing a simple mechanics. For the pitch dynamics we tilt the rotors in such a way that it damps the pendular motion of the vehicle maintaining the upwards position of the propellers. In spite of the complexity of the real prototype's dynamics, a satisfactorily control law performance is observed from the real time experiments. The previous, proves that is possible to deal with strong nonlinear systems by driving each decoupled dynamics.

\section{REFERENCES}

H. Goldstein, C.P. Poole and J.L Safko. Classical Mechanics. Addison-Wesley Publishing Company, Inc., Massachusetts, 1983.

A. Bedford, and W. Fowler. Dynamics. Addison-Wesley Publishing Company, 1989.

P. Castillo, R. Lozano A. Dzul. Modelling and control of mini flying machines. Springer-Verlag, July 2005.

I. Fantoni and R. Lozano. Nonlinear Control for Underactuated Mechanical Systems. Springer-Verlang, 2002.

F. Kendoul, I. Fantoni, and R. Lozano. Modeling and control of a small autonomous aircraft having two tilting rotors. in Proc. 44th IEEE Conf. Decision Control/Euro. Control Conf., Seville, Spain, Dec. 2005, pp. 8144-8149.

G. R. Gress. Using dual propellers as gyroscopes for tiltprop hover control. in Proc. AIAA Biennial Int. Powered Lift Conf. Exhibit, Williamsburg, VA, Nov. 2002, Paper AIAA-2002-5968.

G. R. Gress. A dual-fan VTOL aircraft using opposed lateral tilting for pitch control. in Proc. Amer. Helicopter Soc. 59th Annu. Forum, Phoenix, AZ, May 2003, pp. 2201-2238.

B. Etkin and L. Reid. Dynamics of Flight. J. Wiley $\&$ Sons, Inc., 1991.

B. L. Stevens and F.L. Lewis. Aircraft Control and Simulation 2ed. J. Wiley \& Sons, Inc., 2003.

H. Stone. Aerodynamic Modelling of a Wing-in-Slipstream Tail-Sitter UAV. Biennial International Powered Lift Conference and Exhibit, Williamsburg, Virginia, Nov. $5-7,2002$.

Sussmann H, Sontag E and Yang Y. A General Result on the Stabilization of linear System Using Bounded Control. IEEE TAC 1994 vol (12) 39 pp 2411 - 2425. 